\title{
Improving Data Transmission in Fiber Optics by Detecting Scratches on the Fiber End Face
}

\author{
$1^{\text {st }}$ Nafiseh Vahabi \\ Department of Electronic \\ and Electrical Engineering \\ University College London \\ UK, London \\ uceevah@ucl.ac.uk
}

\author{
$2^{\text {nd }}$ Dongye Yang \\ Department of Electronic \\ and Electrical Engineering \\ University College London \\ UK, London \\ dongye.yang.14@ucl.ac.uk
}

\author{
$3^{\text {rd }}$ David R. Selviah \\ Department of Electronic \\ and Electrical Engineering \\ University College London \\ UK, London \\ d.selviah@ucl.ac.uk
}

\begin{abstract}
Optical fiber sustains scratches, pits and other types of defects on the end face during the polishing process. Hence, fiber end face inspection is a significant process for fiber manufactures when analysing the performance of a fiber. In order to identify the defects present on the fiber end face, a novel model is presented in this paper. Our model combined filtering methods to enhance the contrast of the images so scratches can be successfully detected. However, because the photos have been taken with different gains and exposures, they can not be processed with standard image processing techniques. We developed a method to analyse the defects intensity that could be located under different gains and exposures. We established that the images taken with the high gains and exposures performed well for optical fiber defect recognition.
\end{abstract}

Index Terms-fiber scratch detection, hough transform line detection, image processing, Sobel filter, Gaussian filter

\section{INTRODUCTION}

Optical fiber is the main medium for information transmission in optical communication technology and it is essential to find out the factors that cause degradation of the communication quality. The fiber connector end face inspection is effective and is also critical to the analysis of fiber communications due to the fact that defects in this area proved to be the main reason for network failures [1]. A group of researchers [2] investigated the return loss (RL) performance of optical fiber that is affected by the scratches of optical fiber connections end faces. They defined a new parameter named relative reflectivity. It can be represented as the reflectivity of a fiber divided by the ideal reflectivity of a defect-less fiber interface. A new effective model is established to evaluate the influence of RL by different scratches, which is classified by different size, number, position and relative reflectivity. Through this method, they conclude that the closer the scratches to the centre of the fiber, the greater the degradation. In addition, the scratches located beyond the 25 microns core area led to little effect to the RL [3].

The International Electronics Manufacturing Initiative (iNEMI) team has performed similar research on the Insertion Loss (IL), Return Loss (RL), and the geometric parameters, to develop the defect-less specification of the fiber connector end

The author Nafiseh Vahabi also thanks the UK Government Research council EPSRC for funding her studentship. face [4]. More specifically, they justify the interdependency of the IL to the defect sizes and introduce the Gaussian Weighted Percent Occluded Area (GWPOA) to the intensity distribution of the basic fiber mode. Based on the experimental results, the analysis of an occluded area is a more effective way of the whole blocked area than the count of all the particles number. Although the GWPOA requires further research, it is a useful metric.

The main purpose of this study is providing a judgement for the optical connector end face workmanship, which is a primary cause of network failures. The following work will divide into three parts; firstly, a combination of filtering method applied on images to enhance the contrast of scratches and pits. Secondly, we apply the Hough Transform to the pre processed images to identify the scratches and pits. Finally, in order to get a guideline for the shooting parameters (gain and exposures) relating to the fiber connector end face, we analysed the defects distribution for different shooting parameters.

\section{EXPERIMENTAL DATA}

The images we analyzed are taken from three different types of optical fiber connector end face by using digital camera with different gains and different exposures. The gains are varies from 1, 1.5, 2 and 2.5. The numbers of exposures are: 9.99, $14.97,20.00,24.98,30.00,34.98,39.96,44.98,45.59,49.97$, $54.99,59.97,69.97,74.99$ and 79.98 . Where either gain or exposure increases, the brightness of the image will rise. Two samples of an end face of optical fiber are shown in Fig. 1.
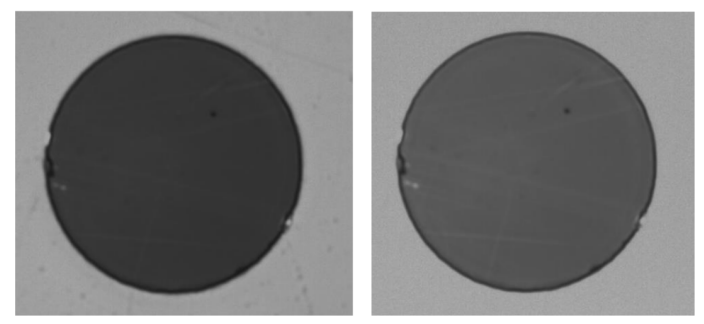

Fig. 1. Original image. Gain 1 and Exposure 47.99 (Left), Gain 1 and Exposure 79.98(Right) 


\section{The Proposed Method}

As these 60 images are shot by different values of gains and exposures, some of the images are too bright or too dark. In order to automatically processing these 60 images, the first step is to unify the background of images to remove the effect of the background. Therefore, images are pre-processed before extracting the defect elements as is described in section III-A.

\section{A. Pre processing images}

Applying filters on images are generally the standard approach to reduce the noise and selecting the filter is application dependant [5], [6]. In our case, Sobel filter was more effective to highlight the areas of high spatial frequency compared to the Canny and Prewitt filter. However, some information is lost in the images after Sobel filter, therefore basic morphological operations are valid to restore more information along the edge areas. The processing was followed by applying the Entropy filter which can characterize the texture of the input image, but it makes the images much more blurry. In order to deal with the blur, the LoG filter is applied to filter the brick out. In the final step, the Gaussian filter used to de-noise the result. The pre-processing model is demonstrated in Fig. 2.

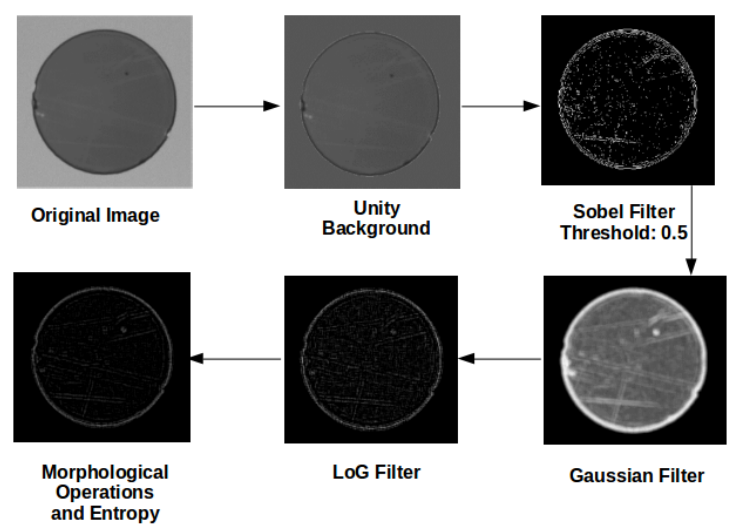

Fig. 2. pre processing steps to detect fiber scratch. (Image: Gain 1 and Exposure 79.98)

\section{B. Defects Recognition Using Hough Transform}

We employed Hough Transform for defect detection as it is [7], [8] a well established method for a line and circle detection in image processing. Before applying Hough Transform, the RGB image was converted to the binary image to remove the noise, improve the defects position accuracy and reduce the computation cost during implementation [9]. Therefore, we set the threshold to 25 and classify white and black pixels as is shown in the image of Fig. 3.

Also, we use Hough Transform to detect the circle component in the core of the fiber to mark the pit. We developed our model using Matlab (R2018b) software. In Matlab, function imfindcircles applies a Circular Hough Transform $(\mathrm{CHT})$ based algorithm to find circles in images. This function is robust to counter the problems of noise, occlusion and varying illumination. The parameter sensitivity is used to define the circular Hough transform accumulator array while the parameter edge sets the threshold to limit the edge pixels in the image. The result shown in Fig. 3 (right hand side) where the green circle marks the pit out.

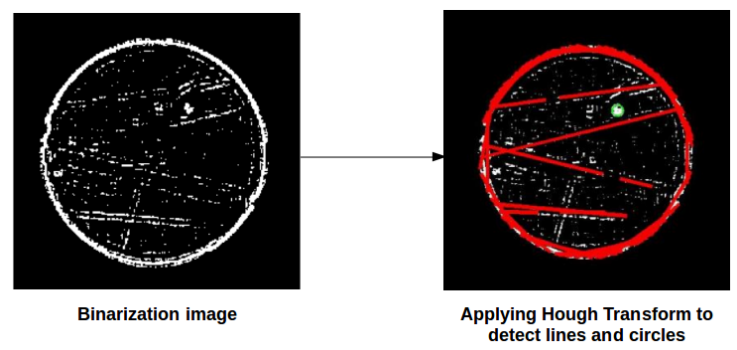

Fig. 3. Image binarization after setting the threshold to 25. (Image: Gain 1 and Exposure 79.98)

\section{Distribution of defects on optical fiber end face}

As the information focus on the fiber centre is to transmit, the standards for the end face quality of a polished fiber optic connector varies according to the region in the fiber. International Electrotechnical Commission (IEC) established standards for setting requirements for allowable surface anomalies, such as scratches and defects, which may affect optical performance [4]. Therefore, analysis of the distribution of the defects in the fiber circle can support the judgement for the quality of fiber connector. We implemented a novel method to analyze the distribution of the defects in order to judge its impact on information transmitted through the optical fiber.

In our proposed method we scan the processed image (Fig. 3 (right)) and sum the value of red pixels which were formed the scratches. The process is started by scanning a circle with very small radius ( 1 micron) and increasing the radius at each iteration. The distribution of red pixels is not uniform in the circle however, the value of red pixel summation is increased by increasing the radius. When the increased radius is large enough, the curve will also stop increasing. The top image of Fig. 4 shows the summation of defect pixel with increasing the radius of scanning in the circle. We also calculated the differential of the curve (bottom image in Fig. 4) with diameter from 0 to 80 micron as it contains more information about scratches.

Intensity curve or R-power curve is an important factor to inspect how the scratches and pits contaminate the optical fiber connector [4]. When the light passes through an optical fiber, the information concentrates in the centre area and that make the optical fibers center area more important than other places. Therefore, the scratches and pits which contaminate the centre area are more vital than the defects existing in other areas. Hence, an intensity cure and its differentiation have been plotted and shown in Fig. 5 top and bottom respectively.

As is shown in top image of Fig. 5, the intensity is decreased as the radius of the optical fiber is increased. When the radius reaches 70 microns the intensity is equal to zero which means the information above this region is not helpful. Also, the 

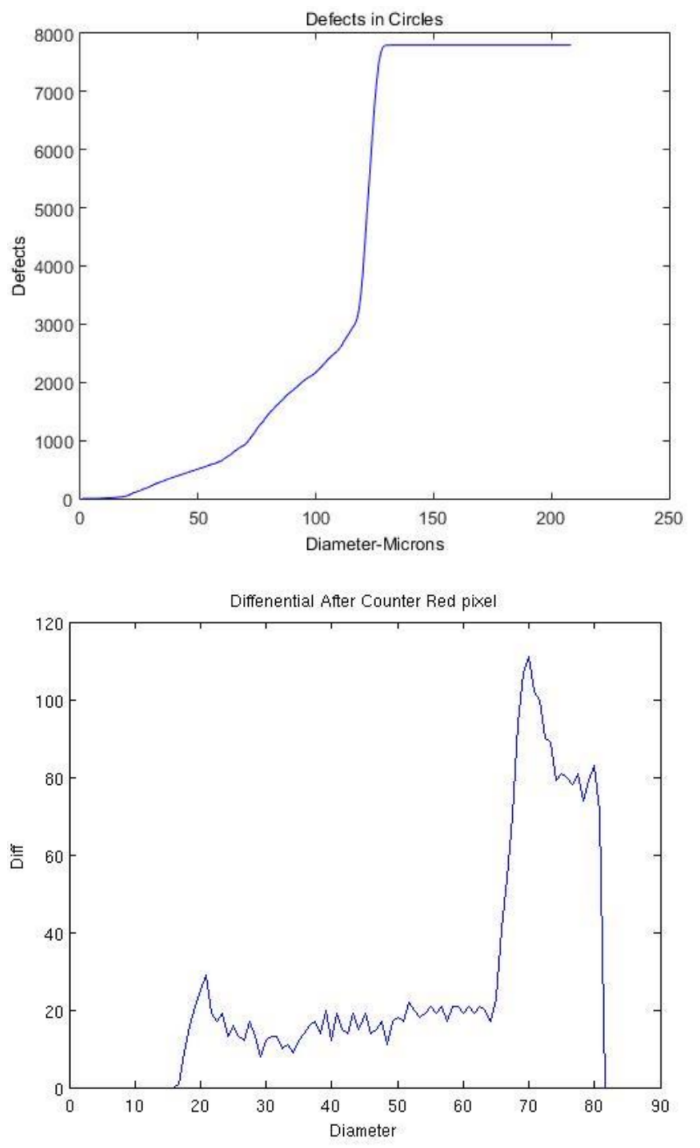

Fig. 4. Defect analysis. The value of red pixels (identified scratches) summation versus diameter of scanning circle is shown in the top image. The bottom image shows the differentiate of the cure in the top image.

bottom image of Fig. 5 shows the differentiation of intensity curve. The curves increases dramatically when the radius reaches 17 microns, which is vital for a high quality optical fiber connector, and then the curve decreases.

\section{Results}

60 images were processed in this study and the result of the analysis is shown in Fig. 6. The $\mathrm{X}$-axis represents the gain of the image which from 1, 1.5, 2 to 2.5. The Y-axis represents the exposure of the image and grows form 9.99 to 79.98 . The $\mathrm{Z}$-axis represents the number of defect pixels in the image.

As it shown in Fig. 6, when the gain and exposure are both low, it is hard to detect the scratches and pit. When the gain and exposure are both high, our defect recognition model performs extremely well. When gain is 2.5 and exposure is 60 , the result around this point all performs well and the trend changes are stable. Therefore, this corner is very appropriate for optical fiber defect recognition and analysis.

\section{CONCLUSION}

In this paper we developed a novel model to analyse the effect of scratches and pits on the end face of optical fiber connector. We processed images by using a combination of
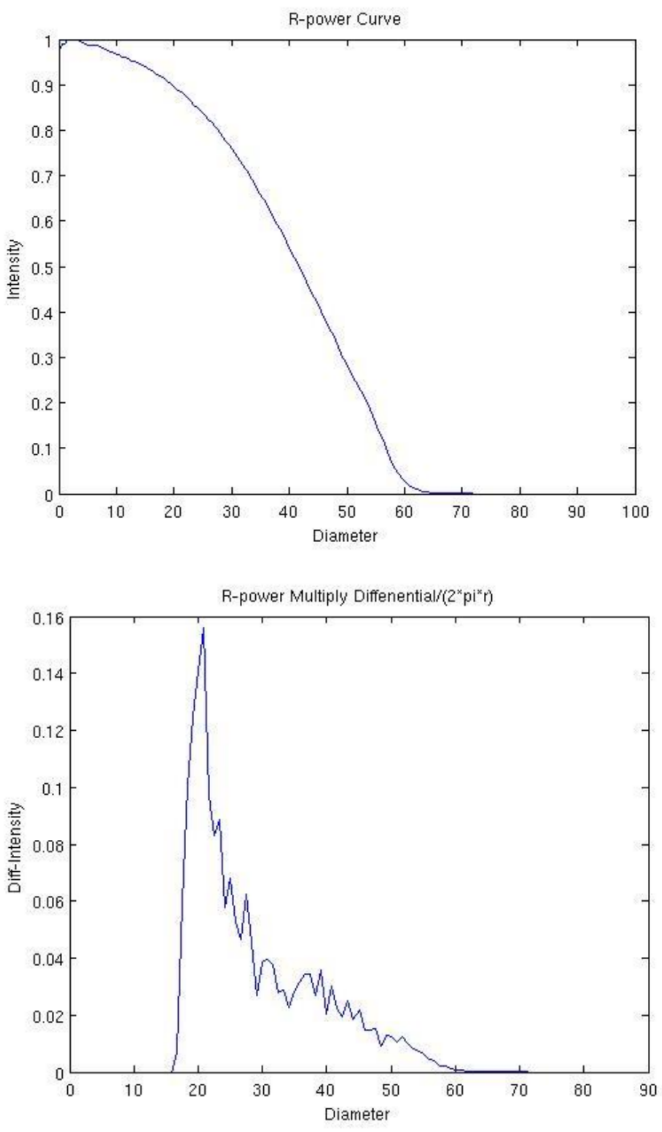

Fig. 5. Intensity curve (top) and intensity curve multiply the differential scaling figure (bottom)

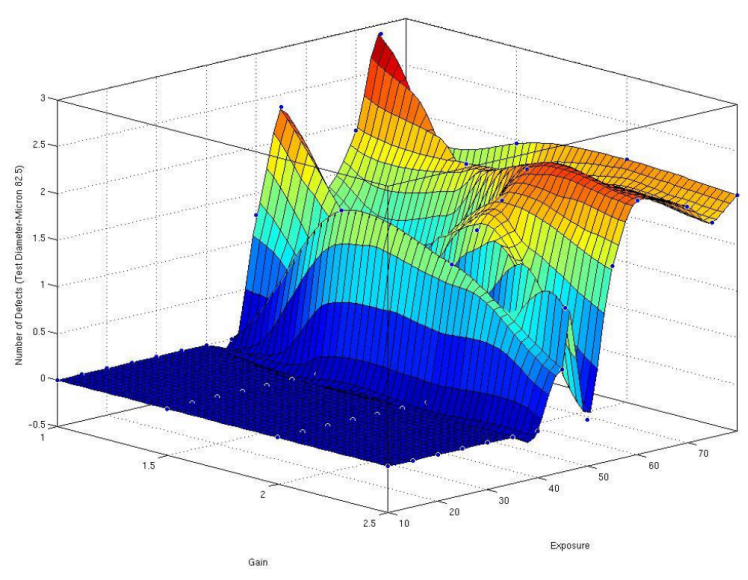

Fig. 6. The number of identified defects were plotted versus gain and exposure.

filters and identified the scratches by applying Hough Transform line detection technique. In addition, we implemented a method to investigate the distribution of the defects on the end face of optical fiber in order to judge its impact on information transmitted through optical fiber. We established the images were taken with the high gain and high exposure 
were performed well for optical fiber defect recognition.

\section{REFERENCES}

[1] W. Mahmood, M. Ozer, and E. Avram, "Surface scratch and pit analysis on optical interfaces," Active and Passive Optical Components for WDM Communication, vol. 4532, pp. 323-330, 2001, International Society for Optics and Photonics.

[2] Z. He, W. Mahmood, E. Sahinci and Y. Pradieu, "Analysis on the effects of fiber end face scratches on return loss performance of optical fiber connectors," Journal of lightwave technology, vol. 22, no. 12, pp. 2749, 2004.

[3] E. Avram, W. Mahmood and M. Ozer, "Quantification of scattering from fiber surface irregularities," Journal of lightwave technology, vol. 20, no. 4, pp. 634, 2002.

[4] T. Berdinskikh, A. Ho, J. Garcia, C. Gleason, S.Y. Huang, J. Kilmer, S. Lytle, T. Mitcheltree, B.J. Roche, H. Tkalec and D. Wilson, "Development of Cleanliness Specifications for Single-Mode Connectors with 1.25 and $2.5 \mathrm{~mm}$ ferrules," Optical Fiber Communication Conference, pp. JThB85, 2006, Optical Society of America.

[5] M. Roushdy, "Comparative study of edge detection algorithms applying on the grayscale noisy image using morphological filter," GVIP journal, vol. 6 , no. 4 , pp. 17-23, 2006.

[6] G. T. Shrivakshan and C. Chandrasekar, "A comparison of various edge detection techniques used in image processing," International Journal of Computer Science Issues (IJCSI), vol. 9, no. 5, pp. 269, 2012.

[7] H.K. Yuen, J. Princen, J. Illingworth and J. Kittler, "Comparative study of Hough transform methods for circle finding," Image and vision computing, vol. 8, no. 1, pp. 71-77, 1990.

[8] J. Illingworth and J. Kittler, "A survey of the Hough transform," Computer vision, graphics, and image processing, vol. 44, no. 1, pp. 87-116, 1988.

[9] J. Sauvola and M. Pietikainen, "Adaptive document image binarization," Pattern recognition, vol. 33, no. 2, pp. 225-236, 2000. 